\title{
The long-range interaction landscape of gene promoters
}

Amartya Sanyal $^{1 *}$, Bryan R. Lajoie ${ }^{1 *}$, Gaurav Jain $^{1}$ \& Job Dekker ${ }^{1}$

The vast non-coding portion of the human genome is full of functional elements and disease-causing regulatory variants. The principles defining the relationships between these elements and distal target genes remain unknown. Promoters and distal elements can engage in looping interactions that have been implicated in gene regulation ${ }^{1}$. Here we have applied chromosome conformation capture carbon copy $\left(5 C^{2}\right)$ to interrogate comprehensively interactions between transcription start sites (TSSs) and distal elements in $1 \%$ of the human genome representing the ENCODE pilot project regions $^{3}$. 5C maps were generated for GM12878, K562 and HeLa-S3 cells and results were integrated with data from the ENCODE consortium ${ }^{4}$. In each cell line we discovered $>1,000$ long-range interactions between promoters and distal sites that include elements resembling enhancers, promoters and CTCFbound sites. We observed significant correlations between gene expression, promoter-enhancer interactions and the presence of enhancer RNAs. Long-range interactions show marked asymmetry with a bias for interactions with elements located $\sim 120$ kilobases upstream of the TSS. Long-range interactions are often not blocked by sites bound by CTCF and cohesin, indicating that many of these sites do not demarcate physically insulated gene domains. Furthermore, only $\sim 7 \%$ of looping interactions are with the nearest gene, indicating that genomic proximity is not a simple predictor for long-range interactions. Finally, promoters and distal elements are engaged in multiple long-range interactions to form complex networks. Our results start to place genes and regulatory elements in three-dimensional context, revealing their functional relationships.

Spatial proximity and specific long-range interactions between genomic elements can be detected using chromosome conformation capture (3C)-based methods ${ }^{5}$. Previous studies have been limited to analysis of single loci ${ }^{5-8}$, interactions that involve a single protein of interest ${ }^{9}$, or to analysis of genome-wide folding of chromosomes at a resolution that cannot detect specific looping interactions between genes and functional elements ${ }^{10}$. To overcome these limitations we previously developed 5C (ref. 2). 5C is a highthroughput adaptation of $3 \mathrm{C}$ and uses pools of reverse and forward $5 \mathrm{C}$ primers to detect long-range interactions between two targeted sets of genomic loci, for example, promoters and distal gene regulatory elements in this study. By targeting a specific part of the genome, $5 \mathrm{C}$ facilitates detection of interactions at single restriction fragment resolution.

To begin to define the principles of long-range gene regulation in the human genome we have used $5 \mathrm{C}$ to map interactions systematically between promoters and distal elements throughout the 44 ENCODE pilot project regions representing $1 \%$ (30 megabases (Mb), Supplementary Table 1) of the genome in three cell lines (Fig. 1a). The ENCODE regions, ranging in size from 500 kilobases $(\mathrm{kb})$ to $1.9 \mathrm{Mb}$, were selected for comprehensive annotation by the ENCODE pilot project ${ }^{11}$. Here we analysed interactions between 628 TSS-containing

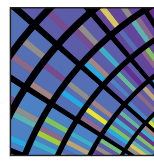

\section{ENCODE}

Encyclopedia of DNA Elements nature.com/encode

tary Tables 2 and 3; see also Methods).

$5 \mathrm{C}$ libraries were generated for two biological replicates of GM12878, K562 and HeLa-S3 (Supplementary Tables 4-6). These cell lines are extensively annotated by the ENCODE consortium ${ }^{3,4}$. 5C interaction frequencies measured between ENCODE regions located on different chromosomes were used to quantify minor variations in interaction detection efficiencies due to technical biases related to $5 \mathrm{C}$ primer efficiency, restriction fragment length, or digestion efficiency. $5 \mathrm{C}$ interaction frequencies were then corrected for these biases (Methods and Supplementary Data).

An example of a 5C long-range interaction map representing TSS-distal fragment interactions along and between 14 ENCODE regions (ENm001-ENm014) is shown in Fig. 1b. 5C detects known general features of spatial chromatin organization. First, interactions within the same ENCODE region are more frequent than those between different ENCODE regions. Within one ENCODE region interaction frequencies are generally higher for pairs of loci located closer together in the linear genome. This inverse relationship between genomic distance and interaction frequency is as expected for a flexible chromatin fibre ${ }^{5,12}$. Second, interactions between ENCODE regions that are located on the same chromosome are more frequent than interactions between regions located on different chromosomes (arrow in Fig. 1b). This is consistent with $4 \mathrm{C}$ and Hi-C analyses ${ }^{6,10}$, and is due to the formation of spatially separated chromosome territories.

$5 \mathrm{C}$ data sets were analysed to identify TSS-distal fragment pairs that interact more frequently than expected, indicating that they are relatively close in space. For each biological replicate we independently determined the average relationship between interaction frequency and genomic distance (solid red lines in Fig. 1c, d). We defined this as the expected interaction frequency. Next we identified interactions that occur significantly more frequently than expected for loci separated by a corresponding genomic distance by transforming $5 \mathrm{C}$ signals into a $z$-score (false discovery rate $(\mathrm{FDR})=1 \%$; Methods). Specific long-range interactions are then defined as pairs of loci that interact significantly more frequently than expected in both replicates. By excluding interactions that are significant in only one replicate, we estimate that only around $10-18 \%$ of the significant long-range interactions identified by our approach might be false positives, as estimated from analysis of interactions in gene desert ENCODE regions (ENr112, ENr113 and ENr313) where no significant long-range interactions were expected (Methods). This application of stringent thresholds probably leads to a higher false-negative rate. Consistently, interaction frequencies that are found to be significant in only one replicate are still significantly elevated in the other replicate as

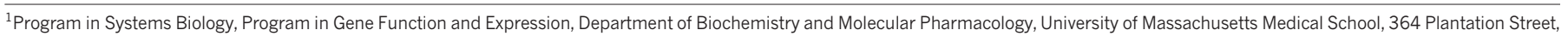
Worcester, Massachusetts 01605-0103, USA.

*These authors contributed equally to this work. 
a
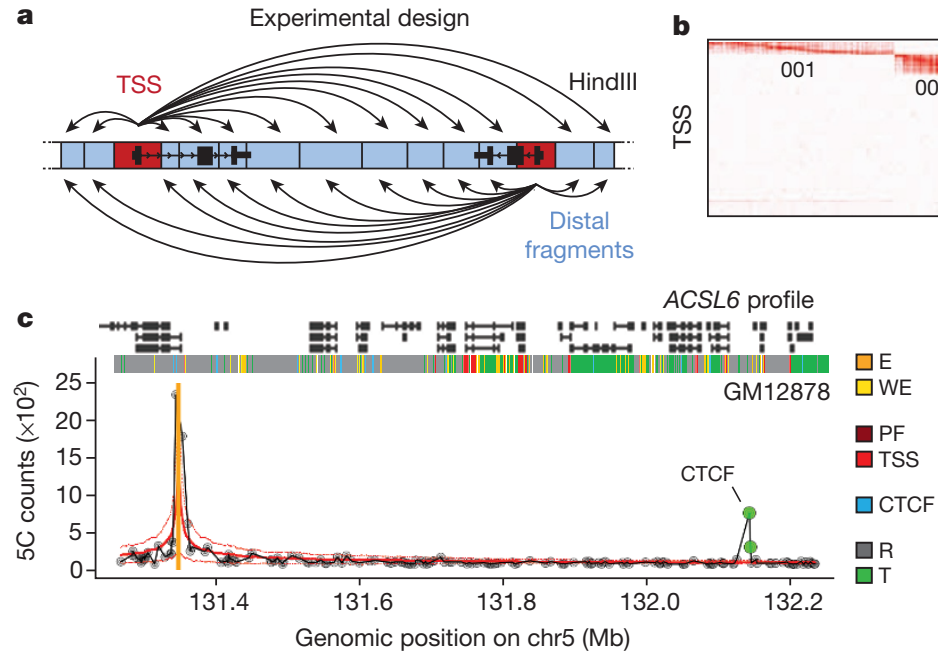

Figure $1 \mid 5 C$ approach to identify looping interactions. a, 5C design ${ }^{28}$. Reverse 5C primers were designed for HindIII fragments that contain a TSS (red; according to the GENCODE $\mathrm{v}^{20}$ ) and forward 5C primers for all other 'distal' HindIII fragments (blue). b, Heat map of all interrogated TSS-distal fragment interactions in 14 ENCODE regions (ENm001-ENm014) in K562 cells. Fragments are displayed in their genomic order. Each dark rectangular area in the heat map denotes interactions within a single ENCODE region whereas remaining areas denote interactions between regions. ENCODE regions that are on the same chromosome show a higher interaction frequency (arrow) than regions that were on different chromosomes. c, d, Examples of 5C interaction profiles for two TSSs indicated by vertical orange bars (left, ACSL6

compared to interactions that are never significant, but are just below the chosen 1\% FDR threshold (Supplementary Fig. 1).

Our analysis correctly identified known interactions between TSSs and their cognate distal regulatory elements, providing validation of the approach (Supplementary Fig. 3). As an example, Fig. 1d shows the $5 \mathrm{C}$ interaction profile in K562 cells for a TSS located in the $\beta$-globin locus. We previously found that this TSS located just downstream of the $\gamma$-globin genes displayed prominent looping interactions with the distal locus control region (LCR) in K562 cells ${ }^{2}$. Our analysis accurately detected these looping interactions (HS3, HS4 and HS5). We identified additional known long-range interactions with DNase I hypersensitive sites (DHSs) near distal CTCF-bound elements (3'HS1 and HS-111 $)^{2,13,14}$. In K562 cells we also detected the known interactions between the $\gamma$-globin gene (HBG1) and the LCR (HS5) and between the $\alpha$-globin genes and three distal regulatory elements including the $\alpha$-globin enhancer HS40, and two CTCF-bound elements (HS46 and HS10), located 40, 46 and $10 \mathrm{~kb}$ upstream of the genes, respectively (Supplementary Fig. 3 and refs 15, 16). The importance of these distal elements in regulating globin gene expression through looping has been extensively documented ${ }^{14,16}$. As expected, these looping interactions in the globin loci were not detected in GM12878 or HeLa-S3 cells that express little or no globin (Supplementary Fig. 3). Additional examples of cell-type-specific TSSdistal element interactions are shown in Supplementary Fig. 4. Furthermore, 5C interaction frequencies are correlated with TSSdistal DHS pairs predicted to be functionally connected based on their highly correlated activity across a large panel of cell lines $\left(P<10^{-13}\right.$, one-sided Mann-Whitney $U$-test ${ }^{17}$ ), providing independent validation of their biological significance.

In each cell line we identified large numbers of statistically significant TSS-distal fragment interactions, of which $\sim 60 \%$ were observed in only one of the three cell lines (Fig. 2a). These data point to intricate cell-type-specific three-dimensional folding of chromatin. 3C-based assays detect specific and functional interactions, for example, TSSs with gene regulatory elements ${ }^{8}$. In addition, the assay will detect 'structural' interactions, for example, close spatial proximity as a result of gene located in ENm002; right, $\gamma$ - $\delta$-globin located in ENm009). The solid red lines show the expected interaction level (Lowess line, Methods); dashed red lines above and below indicate Lowess \pm 1 standard deviation. $5 \mathrm{C}$ signals that are significantly higher than expected in both biological replicates (green circles, $\mathrm{FDR}=1 \%$ ) are considered looping interactions. Interactions that are significant in only one replicate (blue circles) are not considered as a highconfidence $5 \mathrm{C}$ looping interaction. $5 \mathrm{C}$ peak calling detects a long-range interaction between the TSS of ACSL6 and a distal CTCF-bound element in GM12878 cells. The approach identifies the known long-range interactions of $\gamma$ - $\delta$-globin to HS3, HS4, HS5 and HS-111 and several additional DHS and CTCF sites in K562 cells ${ }^{2}$ (labelled).

other nearby specific looping interactions (bystander interactions) or overall higher order folding of the chromatin fibre. To determine which looping interactions involved distal sites that displayed specific chromatin features associated with functional elements, we compared our data with data sets generated by the ENCODE consortium (Fig. 2b and Supplementary Table 7). We found that looping interactions in all cell lines were significantly enriched for distal fragments that are bound by CTCF-a protein known to mediate DNA looping ${ }^{18}$ contain open chromatin (as determined by FAIRE $^{19}$ or DHS mapping ${ }^{17}$ ), and/or contain histones with modifications that are characteristic for active functional elements (H3K4me1, H3K4me2 and $\mathrm{H} 3 \mathrm{~K} 4 \mathrm{me} 3$ ). Long-range interactions are also enriched for $\mathrm{H} 3 \mathrm{~K} 9 \mathrm{ac}$ and $\mathrm{H} 3 \mathrm{~K} 27 \mathrm{ac}$, but are not enriched or significantly depleted for H3K27me3, a mark typically found at inactive or closed chromatin.

To gain more insight into the types of element present in the distal looping fragments, we made use of genome-wide and cell-line-specific segmentation analyses that identified seven distinct chromatin states based on histone modifications, the presence of DHSs and the localization of proteins such as RNA polymerase II and CTCF (ref. 4 and Fig. 2b). These states are: (1) enhancer (E); (2) weak enhancer (WE); (3) TSS; (4) predicted promoter flanking regions (PF); (5) insulator element (CTCF); (6) predicted repressed region (R); and (7) predicted transcribed region (T). The ENCODE consortium tested sets of the E elements in enhancer assays and confirmed that $>50 \%$ display enhancer activity ${ }^{4}$. We found that looping interactions were significantly enriched for distal fragments that contained E, WE and CTCF elements, and the actively transcribed chromatin state (T), but were depleted for the repressed chromatin state (R). We note that some distal looping fragments contained elements classified as TSS or PF, even though they did not contain TSSs as defined by the GENCODE v7 annotation $^{20}$. Possibly, these are yet-to-be-annotated TSSs.

Next, we used the seven-way segmentation data to categorize looping interactions into four broader functional groups (Fig. 2c, Supplementary Fig. 5 and Supplementary Data): those that involve a distal fragment that contains a putative enhancer ('E' (E or WE)), a putative promoter ('P' (TSS or PF)), or a CTCF-bound element 


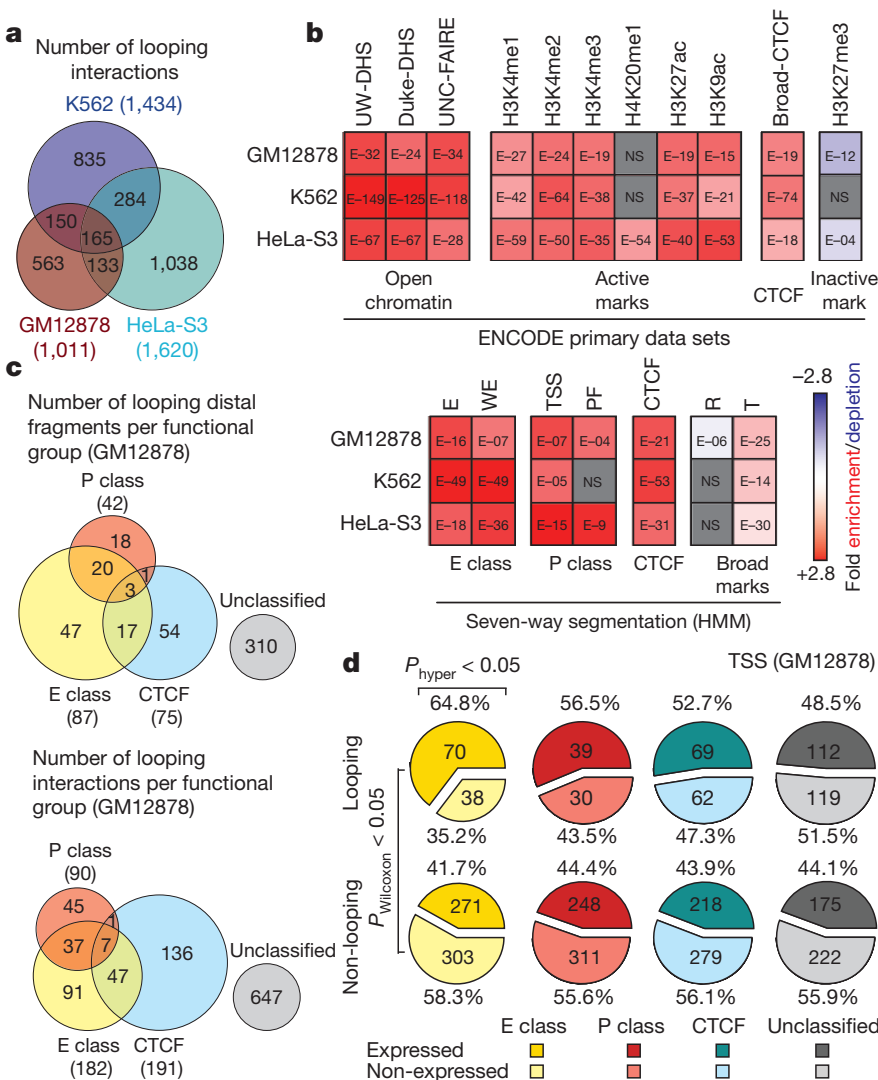

Figure $2 \mid$ Distribution of looping interactions across cell types and their relationship with chromatin features and gene expression. a, Venn diagram showing the number of unique and overlapping looping interactions across three cell types. b, Heat map showing the enrichment/depletion of chromatin features in looping fragments compared to all interrogated fragments based on genome-wide data sets from the ENCODE consortium (Supplementary Table 7). Features include open chromatin (UW-DHS (UW, University of Washington), Duke-DHS and UNC-FAIRE (UNC, University of North Carolina; FAIRE, formaldehyde-assisted isolation of regulatory elements)); active marks (Broad Institute histone H3K4me1/2/3, H4K20me1, H3K27ac, H3K9ac); CTCF (Broad Institute CTCF ChIP peaks); inactive marks (Broad Institute histone H3K27me3); and seven-way segmentation ${ }^{4}$ (based on HMM prediction for indicated cells). We further grouped segmentation categories $\mathrm{E}$ and WE into 'E class', TSS and PF into 'P class', and R and T into 'broad marks'. The colour scale represents the fold enrichment (red) or depletion (blue). The numbers listed inside each box represent $P$ values of the significant $(P<0.05)$ enrichment/depletion for that mark, where (for example) $\mathrm{E}-32$ indicates $\times 10^{-32}$ (NS, not significant, grey; two-tailed hypergeometric test and corrected for multiple testing using Bonferroni). c, Venn diagram showing the number of unique and overlapping looping distal fragments (top) and looping interactions (bottom) among four functional groups in GM12878 cells. Distal fragments are classified into four non-exclusive groups based on the seven-way segmentation. Similarly, TSS-distal fragment interactions are classified based on the functional grouping of the distal fragments. The four functional groups are $\mathrm{E}$ class (yellow), P class (magenta), CTCF (cyan) and unclassified (grey). d, Pie charts showing percentages and numbers of expressed/non-expressed TSSs looping or not looping to a particular group (E, P, CTCF or unclassified; coloured as in c) of distal fragments in GM12878 cells. TSSs with a CAGE value $>0$ are deemed expressed. Significant enrichment for expressed TSSs in the looping or non-looping categories is indicated on top (hypergeometric test; $\left.P_{\text {hyper }}<0.05\right)$. Significant differences in expression levels between TSS in the looping versus the non-looping category is indicated on the left (Wilcoxon signed-rank test; $\left.P_{\text {Wilcoxon }}<0.05\right)$.

(CTCF). The final class contains interactions with fragments that do not contain any of these three types of element, although they do contain T and R states (' $U$ ', unclassified). The last class is relatively large but is still significantly enriched in features that are characteristic for active functional elements such as $\mathrm{H} 3 \mathrm{~K} 4 \mathrm{me} 1$, and over $60 \%$ of the unclassified fragments contain chromatin features found at active chromatin elements (Supplementary Fig. 7). Thus, these are not simply noise or false positives, but are probably the result of the conservative segmentation approach.

We found that TSS-E and TSS-P interactions are more cell-type specific than TSS-CTCF interactions: for the TSS-E and TSS-P categories, the ratio of interactions that is seen in only one cell line versus more than one cell line is $\sim 4: 1$, whereas it is close to $\sim 1: 1$ for the TSS-CTCF category (Supplementary Fig. 5). The cell-type-specific activity of some of these E elements was confirmed using transient reporter assays (Supplementary Fig. 10). Next, we determined whether looping of a TSS to any of the four categories of chromatin states is correlated with transcription. We used CAGE expression data ${ }^{21}$ to assign an expression level to each TSS. We found that looping interactions with fragments containing enhancer-like E elements were significantly enriched for those that involved expressed TSSs (Fig. 2d and Supplementary Fig. 6). In addition, the subset of TSSs that interact with fragments containing E elements was significantly more highly expressed compared to TSSs that do not interact with $\mathrm{E}$ elements. Interactions with other classes of element (CTCF, P and U) are significantly enriched for actively expressed genes in some, but not all, cell lines (Supplementary Fig. 6).

Active enhancers often express enhancer RNAs ${ }^{22}$. We used a comprehensive enhancer RNA data set generated by the ENCODE consortium to determine whether TSSs preferentially interact with active enhancer-like elements ${ }^{23}$. We found that E elements that are looping to TSSs are significantly more likely to express enhancer RNAs than E elements that are not looping $\left(P<5 \times 10^{-5}\right.$, hypergeometric test, Supplementary Fig. 10). We conclude that looping interactions preferentially involve active enhancer-like elements.

Next we analysed the distribution of long-range interactions upstream and downstream of TSSs. To generate this landscape of looping interactions we aligned all TSSs and calculated the average number of interactions that a TSS has with each class of distal element at increasing genomic distances upstream and downstream of the TSS. Figure $3 \mathrm{a}$ shows the resulting average long-range interaction profile across all three cell lines (similar results were obtained when each of the cell lines was analysed separately; Supplementary Fig. 8). Notably, we found that the long-range interaction landscape is asymmetric, with interactions of E, P and CTCF classes peaking around $120 \mathrm{~kb}$ upstream of the TSS. This asymmetry of interactions reveals an unanticipated directionality in long-range interactions with TSSs. This may indicate the presence of topological constraints imposed by the mechanism by which such interactions regulate target promoters. No such bias was observed for the set of unclassified elements, or for the complete set of interrogated interactions (Fig. 3a). Interestingly, previous analyses showed that conserved non-coding elements are also often found within similar distances of target genes ${ }^{24}$. Third, when we analysed expressed TSSs and nonexpressed TSSs separately, we found that both have a similar interaction landscape but that expressed TSSs tend to have more interactions, especially with the E, P and CTCF classes. We cannot rule out the possibility that some TSSs classified as non-expressed based on the absence of CAGE tags are actually expressed at low levels.

Next we explored whether the relative order of elements in the genome affects which long-range interactions occur. It is often assumed that distal elements such as enhancers target the nearest TSS. Only 7\% of the looping interactions are between an element and the nearest TSS (Fig. 3b). This number goes up to $22 \%$ when only active TSSs are included. Similarly, $27 \%$ of the distal elements have an interaction with the nearest TSS, and $47 \%$ of elements have interactions with the nearest expressed TSS. Thus, when predicting TSS-distal element interactions, choosing the nearest (active) gene is often not correct.

It has been suggested that CTCF sites located between an enhancer and a TSS may prevent enhancer-promoter interactions ${ }^{18,25}$, although in individual cases interactions over such sites have been observed ${ }^{14,26}$. 


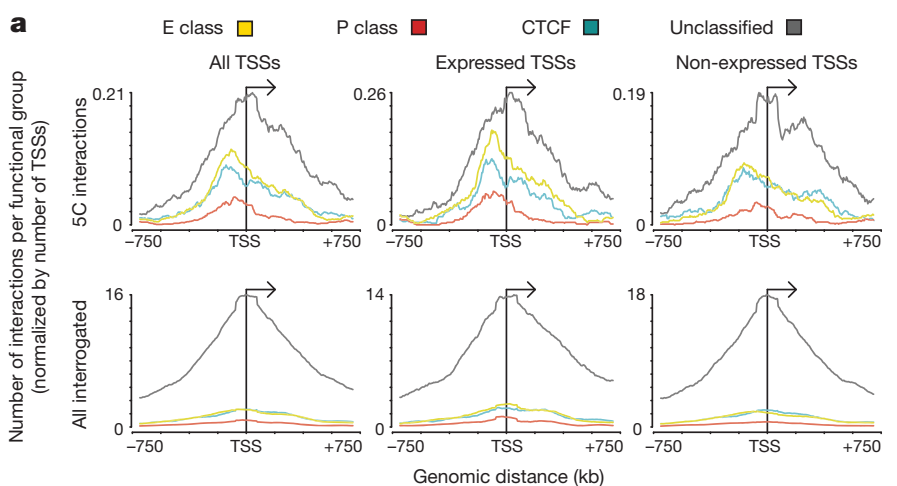

b c
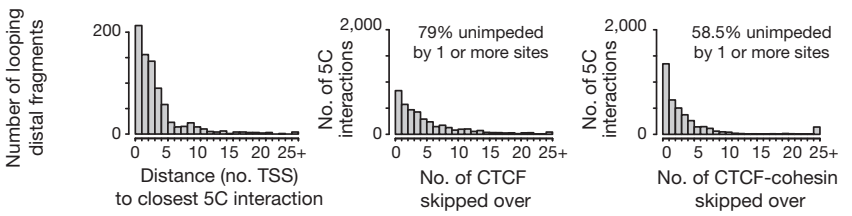

Figure 3 Looping landscape of TSSs to distal fragments. a, Composite profile of average number of group-specific looping interactions upstream and downstream of TSSs on the basis of combined 5C interaction data from the three cell lines. The top panel shows the average looping profiles of all TSSs (left), of expressed TSSs (middle) and of non-expressed TSSs (right). The bottom set of plots shows the corresponding profiles of all interrogated TSSdistal element interactions (left), of expressed TSSs (middle) and of nonexpressed TSSs (right). All the interaction data for a particular group for all three cell lines are binned with a sliding window of $150 \mathrm{~kb}$ (step size of $5 \mathrm{~kb}$ ) and normalized for the number of TSSs. $\mathbf{b}$, Histogram showing the number of distal fragments that are involved in looping with their target promoters skipping $0,1,2, \ldots, 25$ (and above) TSSs. c, Histogram showing the number of looping interactions that skip over $0,1,2, \ldots, 25$ (and above) restriction fragments bound by either CTCF (left) or by both CTCF and RAD21 (cohesin; right). In b and $\mathbf{c}$ combined results for all three cell lines are plotted and values above 24 on the $x$ axis are added and grouped as 25+. Percentage of looping interactions that skip $\geq 1$ CTCF (left) or CTCF plus cohesin (right) are indicated on top.

To address this question we determined the frequency of identified long-range interactions between a TSS and a distal element that skip over one or more sites bound by CTCF. We found that $79 \%$ of longrange interactions are unimpeded by the presence of one or more CTCF-bound sites (Fig. 3c). Thus, the presence of a CTCF-bound site does not block physical long-range interactions. It has been reported that CTCF acts in conjunction with the cohesin complex to block promoter-enhancer interactions ${ }^{27}$. We found that $58 \%$ of looping interactions skip sites co-bound by CTCF and cohesin (Fig. 3c). We obtained similar results when the different categories of long-range interaction (TSS-E, TSS-P, TSS-CTCF and TSS-U) were analysed separately. Possibly, additional factors need to be recruited to CTCFbound sites to acquire interaction-blocking activity.

The large number of long-range interactions that we discovered indicate that distal elements and TSSs are each engaged in multiple long-range interactions. To characterize this phenomenon in more detail we determined the interaction degree of TSSs and distal fragments. We found that $\sim 50 \%$ of TSSs display one or more long-range interaction, with some interacting with as many as 20 distal fragments (Fig. 4a). Expressed TSSs interact with slightly more fragments as compared to non-expressed TSSs (the mean for GM12878 is 1.88 versus 1.37 , or 3.88 versus 3.25 when including only those TSSs with at least one interaction). Out of all distal fragments interrogated, $\sim 10 \%$ interacted with one or more TSS, with some interacting with more than 10 (mean of 2.15 (for GM12878) when including only those distal fragments with at least one interaction). The degree distribution of the four categories of distal elements was very similar (Supplementary Fig. 9).

Figure $4 \mathrm{~b}$ shows an example of the complex long-range interaction networks formed by TSSs and distal fragments in the ENr132 region in
K562 cells. It is unlikely that these interactions can all occur at the same time in the same cell, which is indicative of significant cell-to-cell variation. The data indicate that gene-element interactions are not exclusively one-to-one, and suggest that multiple genes and distal elements can assemble in larger clusters, as proposed for the $\beta$-globin locus $^{14}$.

Our data provide new insights into the landscape of chromatin looping that bring genes and distant elements in close spatial proximity. In addition to generating a rich data set reflecting specific gene-element interactions, the average interaction profile of TSSs with surrounding chromatin reveals several general principles regarding the asymmetric relationships between genomic distance, the order of elements, and the formation of looping interactions. The bias for upstream interactions may indicate that the protein complexes on many TSSs may be asymmetric and may preferentially interact on one side with enhancer-protein complexes. It is also possible that the asymmetry of the long-range interaction landscape reflects a potential preference of looping to elements that are located in intergenic non-transcribed regions. Furthermore, although these average long-range interaction landscapes may facilitate computational prediction of long-range interactions throughout the genome, the fact that interactions skip genes and CTCF/cohesin sites indicates that additional mechanisms for target selection and gene insulation exist.

Although conventional 3C may still be the method of choice to study the folding of individual loci, the $5 \mathrm{C}$ design strategy and data analysis methods applied here may provide a general approach for systematically

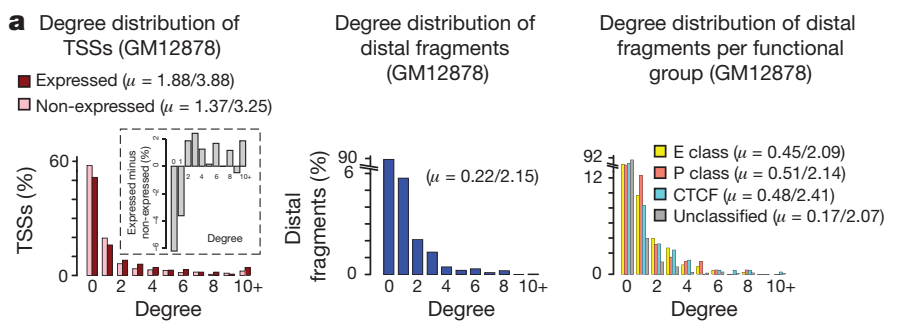

b $\square$ Eclass $\square$ P class $\square$ CTCF $\square$ Unclassified $\square$ P class + CTCF $\square$ E class $+C T C F$ - Distal fragments OTSS expressed •TSS non-expressed

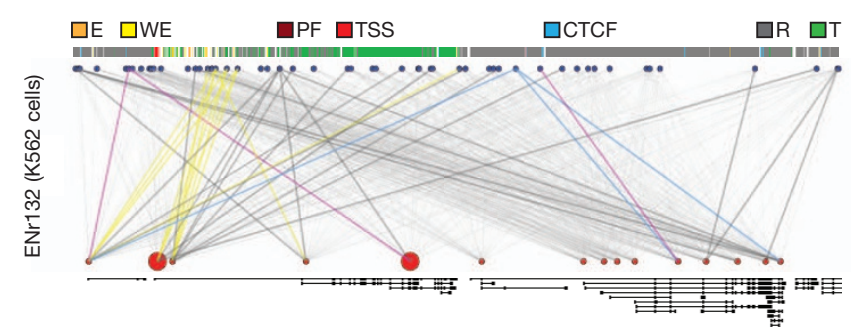

Figure $4 \mid$ Networks of looping interactions. a, Histogram showing the number of TSSs (left, red) or distal fragments (middle, blue) in percentages that are involved in $0,1,2, \ldots 10$ (and above) looping interactions (degree, $x$ axis) in GM12878 cells. All of the values for degrees that are $>9$ are grouped under degree $10+$. The dark red bars represent the percentages of looping TSSs that are expressed whereas light red bars represent the percentages of looping TSSs that are not expressed. Inset: the difference in percentage between looping TSSs that are expressed and not expressed for each degree is shown. The right panel shows the degree distribution for each functional group of distal fragments. The average degrees (mean, $\mu$ ) for TSSs and distal fragments are indicated. The first value is the mean degree considering all the TSS/distal fragments (looping plus non-looping), whereas the second value is the mean degree of looping TSS/ distal fragments (excluding degree $=0$ ). $\mathbf{b}$, Web plot showing the long-range looping interactions in the ENr132 region in K562 cells. The interrogated distal fragments (blue circles) and the TSSs (red circles) are positioned according to genomic coordinates and the GENCODE v7 gene annotation is indicated. The size of the red circles indicates whether that TSS is expressed (large circles) or not expressed (small circles). The thin grey lines show all the interactions that were interrogated. The coloured lines show significant looping interactions between TSSs and distal fragments of a particular group. 
mapping gene-element interactions for large gene sets. With further development of $3 \mathrm{C}$ technology and increases in sequencing capacity, similar high-resolution studies should become feasible to map specific long-range interactions throughout the genome, which may uncover additional principles that guide chromatin looping. Such insights will also be critical for interpreting genome-wide association studies that often identify regions with regulatory elements but not their distally located target genes. Co-published ENCODE-related papers can be explored online via the Nature ENCODE explorer (http://www. nature.com/ENCODE), a specially designed visualization tool that allows users to access the linked papers and investigate topics that are discussed in multiple papers via thematically organized threads.

\section{METHODS SUMMARY}

5C was performed using two pools of 5C primers: one for ENm001-ENm014 and ENr313, and one pool for all 30 randomly picked ENCODE regions (ENr111ENr334) ${ }^{11}$ (Supplementary Tables 2 and 3). 5C libraries (two biological replicates per cell line) were sequenced on an Illumina GAIIx platform and sequence reads were mapped using Novoalign (http://www.novocraft.com), as described ${ }^{15}$. Interaction data for each experiment are available in GEO (accession number GSE39510). Statistically significant pair-wise interactions were identified (Methods) by converting each $5 \mathrm{C}$ signal into a $z$-score using the average $5 \mathrm{C}$ signal distribution versus genomic distance as a background estimate. Significant interactions (1\% FDR) observed in both biological replicates were considered looping interactions. 5C looping interactions were compared to a variety of genome-wide data sets generated by the ENCODE consortium ${ }^{4}$ (Supplementary Table 7).

Full Methods and any associated references are available in the online version of the paper.

\section{Received 9 December 2011; accepted 1 June 2012.}

1. Dekker, J. Gene regulation in the third dimension. Science 319, 1793-1794 (2008)

2. Dostie, J. et al. Chromosome conformation capture carbon copy (5C): A massively parallel solution for mapping interactions between genomic elements. Genome Res. 16, 1299-1309 (2006)

3. ENCODE Project Consortium. A user's guide to the encyclopedia of DNA elements (ENCODE). PLoS Biol. 9, e1001046 (2011).

4. ENCODE Project Consorium. An integrated encyclopedia of DNA elements in the human genome. Nature http://dx.doi.org/10.1038/nature11247 (this issue).

5. Dekker, J., Rippe, K., Dekker, M. \& Kleckner, N. Capturing chromosome conformation. Science 295, 1306-1311 (2002).

6. Simonis, M. et al. Nuclear organization of active and inactive chromatin domains uncovered by chromosome conformation capture-on-chip (4C). Nature Genet. $\mathbf{3 8}$ 1348-1354 (2006).

7. Zhao, Z. et al. Circular chromosome conformation capture (4C) uncovers extensive networks of epigenetically regulated intra- and interchromosomal interactions. Nature Genet. 38, 1341-1347 (2006).

8. Miele, A. \& Dekker, J. Long-range chromosomal interactions and gene regulation. Mol. Biosyst. 4, 1046-1057 (2008).

9. Fullwood, M. J. et al. An oestrogen-receptor- $\alpha$-bound human chromatin interactome. Nature 462, 58-64 (2009).

10. Lieberman-Aiden, E. et al. Comprehensive mapping of long-range interactions reveals folding principles of the human genome. Science 326, 289-293 (2009).

11. ENCODE Project Consortium. Identification and analysis of functional elements in $1 \%$ of the human genome by the ENCODE pilot project. Nature $447,799-816$ (2007).
12. Gheld of, N., Tabuchi, T. M. \& Dekker, J. The active FMR1 promoter is associated with a large domain of altered chromatin conformation with embedded local histone modifications. Proc. Natl Acad. Sci. USA 103, 12463-12468 (2006).

13. Palstra, R. J. et al. The $\beta$-globin nuclear compartment in development and erythroid differentiation. Nature Genet. 35, 190-194 (2003).

14. Tolhuis, B., Palstra, R. J., Splinter, E., Grosveld, F. \& de Laat, W. Looping and interaction between hypersensitive sites in the active $\beta$-globin locus. Mol. Cell 10 1453-1465 (2002)

15. Baù, D. et al. The three-dimensional folding of the $\alpha$-globin gene domain reveals formation of chromatin globules. Nature Struct. Mol. Biol. 18, 107-114 (2011)

16. Vernimmen, D., De Gobbi, M., Sloane-Stanley, J. A., Wood, W. G. \& Higgs, D. R. Long range chromosomal interactions regulate the timing of the transition between poised and active gene expression. EMBO J. 26, 2041-2051 (2007).

17. Thurman, R. E. et al. The accessible chromatin landscape of the human genome Nature http://dx.doi.org/10.1038/nature11232 (this issue).

18. Phillips, J. E. \& Corces, V. G. CTCF: master weaver of the genome. Cell 137, 1194-1211 (2009)

19. Song, L. et al. Open chromatin defined by DNasel and FAIRE identifies regulatory elements that shape cell-type identity. Genome Res. 21, 1757-1767 (2011)

20. Harrow, J. et al. GENCODE: The reference human genome annotation for the ENCODE project. Genome Res. http://dx.doi.org/10.1101/gr.135350.111 (2012).

21. Dong, X. et al. Correlating histone modifications and gene expression. Genome Biol.. (in the press).

22. Kim, T. K. et al. Widespread transcription at neuronal activity-regulated enhancers. Nature 465, 182-187 (2010)

23. Djebali, S. et al. Landscape of transcription in human cell lines. Nature http:// dx.doi.org/10.1038/nature11233 (this issue).

24. Vavouri, T., McEwen, G. K., Woolfe, A., Gilks, W. R. \& Elgar, G. Defining a genomic radius for long-range enhancer action: duplicated conserved non-coding elements hold the key. Trends Genet. 22, 5-10 (2006).

25. Wallace, J. A. \& Felsenfeld, G. We gather together: insulators and genome organization. Curr. Opin. Genet. Dev. 17, 400-407 (2007)

26. Kurukuti, S. et al. CTCF binding at the $\mathrm{H} 19$ imprinting control region mediates maternally inherited higher-order chromatin conformation to restrict enhancer access to lgf2. Proc. Natl Acad. Sci. USA 103, 10684-10689 (2006).

27. Wendt, K. S. et al. Cohesin mediates transcriptional insulation by CCCTC-binding factor. Nature 451, 796-801 (2008)

28. Lajoie, B. R., van Berkum, N. L., Sanyal, A. \& Dekker, J. My5C: web tools for chromosome conformation capture studies. Nature Methods 6, 690-691 (2009)

Supplementary Information is available in the online version of the paper.

Acknowledgements We thank the University of Massachusetts Medical School Deep Sequencing core for sequencing $5 \mathrm{C}$ libraries, and $\mathrm{R}$. Thurman and J. Stamatoyannopoulos for discussion and help with peak calling analysis. We thank M. Walhout and members of the Dekker laboratory for discussions. This work was supported by grants from the National Institutes of Health, National Human Genome Research Institute (HG003143 and HG003143-06S1) and a W.M Keck Foundation Distinguished Young scholar in Medical Research award to J.D.

Author Contributions J.D. conceived the project. A.S. performed all experiments. B.R.L. designed $5 \mathrm{C}$ experiments, and built the data analysis and visualization pipelines. B.R.L., A.S., G.J. and J.D. analysed the data and wrote the paper.

Author Information All data are publicly available at GEO (accession number GSE39510). 5C data has also been deposited in the public UCSC ENCODE database (http://encodeproject.org/ENCODE/). 5C data can be found at http:// hgdownload.cse.ucsc.edu/goldenPath/hg19/encodeDCC/ wgEncodeUmassDekker5C/. Reprints and permissions information is available at www.nature.com/reprints. This paper is distributed under the terms of the Creative Commons Attribution-Non-Commercial-Share Alike licence, and the online version of the paper is freely available to all readers. The authors declare no competing financial interests. Readers are welcome to comment on the online version of the paper. Correspondence and requests for materials should be addressed to J.D. (job.dekker@umassmed.edu). 


\section{METHODS}

Cell growth conditions. GM12878 lymphoblastoid cells were procured from Coriell Cell Repositories and grown in RPMI 1640 medium supplemented with $2 \mathrm{mM}$ L-glutamine, $15 \%$ fetal bovine serum (FBS) and antibiotic (1\% penicillinstreptomycin). K562 (CCL-243), a CML cell line, and HeLa-S3 (CCL2.2), a cervical carcinoma cell line, were obtained from American Type Culture Collection (ATCC). K562 cells were cultured in similar media as GM12878 cells except with $10 \%$ FBS, whereas HeLa-S3 cells were maintained in ATCC recommended F-12K medium (Kaighn's modification of Ham's F-12 medium) with $10 \%$ FBS and $1 \%$ penicillin-streptomycin. The culture densities and conditions were maintained as per recommendations of the repositories.

Formaldehyde crosslinking. For suspension cells (GM12878, K562) a total of $1 \times 10^{8}$ freshly growing cells were centrifuged at $100 \mathrm{~g}$ for $5 \mathrm{~min}$. Cell pellets were re-suspended in $45 \mathrm{ml}$ of respective growth medium in a 50-ml Falcon tube. Cells were fixed by addition of $1.25 \mathrm{ml}$ of $37 \%$ formaldehyde (final concentration of formaldehyde $1 \%$ ). The cell suspension was gently mixed by inverting the tube up and down 4-6 times at room temperature and the tubes were rotated on an end-toend shaker for exactly $10 \mathrm{~min}$. Crosslinking was stopped by addition of $2.5 \mathrm{M}$ glycine (final concentration $125 \mathrm{mM}$ ) and cell suspensions were incubated at room temperature for $15 \mathrm{~min}$ using an end-to-end shaker. The crosslinked cells were then pelleted at $100 \mathrm{~g}$ for $5 \mathrm{~min}$ and the cell pellet was stored at $-80^{\circ} \mathrm{C}$. For HeLa-S3 cells, the adherent cells were first trypsinized and then the crosslinking was performed as described above.

5C analysis. 5C analysis was carried out as previously described ${ }^{2,15}$ for the 44 ENCODE Pilot regions (ENCODE manual (ENm) and ENCODE random $(\mathrm{ENr}))$. The chromosomal position and coordinates of the regions as per the February 2009 GRCh37/hg19 human genome assembly are listed in Supplementary Table 1 . The $5 \mathrm{C}$ experiment is designed to interrogate looping interactions between HindIII fragments containing transcription start sites (TSSs) and any other HindIII restriction fragment (distal fragments) in the ENCODE pilot regions.

5C primer design. 5C primers were designed at HindIII restriction sites (AAGCTT) using 5C primer design tools previously developed and made available online at My5C website (http://my5C.umassmed.edu) ${ }^{28}$. Reverse $5 \mathrm{C}$ primers were designed for HindIII restriction fragments overlapping a known TSS from GENCODE transcripts, or overlapping a start site as experimentally determined by CAGE tag data of the ENCODE pilot project (Supplementary Table 2). Forward 5C primers were designed for the remaining HindIII restriction fragments (Supplementary Table 3). For ENCODE regions that do not contain any TSS according to gene annotation in 2008 (ENr112, ENr113, ENr311 and ENr313), we used an alternative primer design. For these regions an alternating design of forward and reverse $5 \mathrm{C}$ primers was used in which forward and reverse primers are designed for alternating restriction fragments ${ }^{2}$. Note that ENr311 contains genes according to 2011 GENCODE v7 annotation ${ }^{20}$. Primers were excluded for highly repetitive sequences that prevented the design of a sufficiently unique $5 \mathrm{C}$ primer. Primers settings were as described before ${ }^{15}$ : U-BLAST, 3; S-BLAST, 130; 15-MER, 1,320; MIN_FSIZE, 40; MAX_FSIZE, 50,000; OPT_TM, 65; OPT_PSIZE, 40 . The 5C primers contained up to 40 bases that were specific for the corresponding restriction fragment. If a shorter sequence was sufficient to obtain a predicted annealing temperature of $65^{\circ} \mathrm{C}$, that shorter sequence was used, and random sequence was added to make a total of 40 bases. All of the $5 \mathrm{C}$ primers have an extension of universal tail sequences at the $5^{\prime}$ end for forward $5 \mathrm{C}$ primers and at the $3^{\prime}$ end for reverse $5 \mathrm{C}$ primers. DNA sequence of the universal tails of forward primers was 5'-CCTCTCTATGGGCAGTCGGTGAT-3'; DNA sequence for the universal tails of reverse primers was $5^{\prime}$-AGAGAATGAGG AACCCGGGGCAG-3'. A six-base barcode was included between the specific sequence of the primers and the universal tail to aid in mapping of the highthroughput short sequencing reads. The length of each primer was $69 \mathrm{bp}$. In total, 981 reverse primers and 5,321 forward primers were designed (corresponding to $\sim 77.1 \%(6,302$ of 8,174$)$ of all HindIII fragments in the 44 ENCODE regions).

Generation of 5C libraries. 3C was performed with HindIII restriction enzyme as previously described ${ }^{15,29}$ for GM12878, K562 and HeLa-S3 cells separately with two biological replicates for each cell line. The $3 \mathrm{C}$ libraries were then interrogated by $5 \mathrm{C}$. The $44 \mathrm{ENCODE}$ regions were analysed in two groups using two separate $5 \mathrm{C}$ primer pools. The first group $(\mathrm{ENm})$ contained the manually picked ENCODE regions ENm001-ENm014 and ENr313. The second group (ENr) contained the 30 randomly picked ENCODE regions. The two $5 \mathrm{C}$ primer pools were made by pooling $5 \mathrm{C}$ primers for interrogating long-range interactions in the two groups of ENCODE regions. In these pools each primer was present at a final concentration of $0.5 \mathrm{fmol} \mu \mathrm{l}^{-1}$

The primer pool for the ENm group contained a total of 3,150 primers (476 reverse $5 \mathrm{C}$ primers and 2,674 forward $5 \mathrm{C}$ primers). This primer pool allows interrogation of a total of $1,272,824$ interactions. Of these, 83,427 interactions were between fragments that were both located in the same ENCODE region The primer pool for the ENr group contained a total of 3,152 primers ( 505 reverse $5 \mathrm{C}$ primers and 2,647 forward 5C primers). This primer pool allows interrogation of a total of 1,336,735 interactions. Of these, 34,859 interactions were between fragments that were both located in the same ENCODE region.

$5 \mathrm{C}$ was performed in 10-15 reactions each containing an amount of 3C library that represents 200,000 genome equivalents and $0.5 \mathrm{fmol}$ of each primer. The multiplex annealing reaction was performed overnight at $55^{\circ} \mathrm{C}$. Pairs of annealed $5 \mathrm{C}$ primers were ligated at the same temperature using Taq DNA ligase for $1 \mathrm{~h}$ Ligated $5 \mathrm{C}$ primer pairs, which represent a specific ligation junction in the $3 \mathrm{C}$ library and thus a long-range interaction between the two corresponding loci, were then amplified using 28 cycles of PCR with universal tail primers that recognize the common tails of the 5C forward and reverse primers. At least four separate amplification reactions were carried out for each of 10-15 annealing reactions described above and all the PCR products were pooled together. This pool constitutes the 5C library. The libraries were concentrated using Qiaquick PCR purification kit and a $3^{\prime}$-A tailing reaction was done using dATP and Taq DNA polymerase in the presence of $1 \times$ standard Taq buffer (NEB) at $72{ }^{\circ} \mathrm{C}$ for $30 \mathrm{~min}$

To facilitate Illumina paired-end DNA sequence analysis of $5 \mathrm{C}$ libraries, Illumina paired-end adaptor oligonucleotides (Illumina) were ligated to the $5 \mathrm{C}$ library using the Illumina PE protocol. The linkered 5C library was then amplified by PCR (17 or 18 cycles, with Phusion High Fidelity DNA polymerase) using Illumina PCR primer PE 1.0 and 2.0. The 5C library was gel purified and sequenced on the Illumina GAIIx platform, generating 36-bp paired-end reads. 5C read mapping. Sequencing data was obtained from an Illumina GAIIx machine and was processed through a custom pipeline to map and assemble $5 \mathrm{C}$ interactions. We used 36-bp paired-end reads to sequence all 5C libraries. Owing to sequencing efficiency, some $5 \mathrm{Clibraries} \mathrm{were} \mathrm{re-sequenced} \mathrm{as} \mathrm{many} \mathrm{as} \mathrm{ten} \mathrm{times}$ to obtain the required read depth for our analysis.

The fastQ files were taken directly from the Illumina GAIIx and fed into our in-house 5C mapping pipeline. Each side of the paired end read was independently mapped to a pseudo-genome of all possible $5 \mathrm{C}$ primer sequences using the novoalign mapping algorithm (V2.05 http://novocraft.com). The default alignment settings for novoalign were used. After mapping, if both of the paired-end reads could be uniquely mapped to a $5 \mathrm{C}$ primer, a $5 \mathrm{C}$ interaction was assembled. Invalid interactions between the same primer or between primers of the same type were removed as these would represent a mapping artefact or an issue with the $5 \mathrm{C}$ technique. The number of invalid interactions detected across all libraries was $<0.01 \%$, which would be expected if solely due to random mapping errors.

Statistics regarding the $5 \mathrm{C}$ library quality, mapping efficiency, etc. can be found in Supplementary Table 4. Because it is only necessary to map the paired-end reads to the list of all possible 5C primers rather than to the entire genome, a higher percentage of mapped/usable reads can be achieved. We found that $>90 \%$ of all paired-end reads (after Illumina chastity filtering) can be uniquely mapped to a single 5C interaction. For libraries where more than one lane was used to achieve adequate sequence depth, the interactions from each lane were summed to produce the complete $5 \mathrm{C}$ interaction data set. A table summarizing the read depth of each 5C library can be found in Supplementary Table 5. Pearson correlation coefficients between the biological replicates can be found in Supplementary Table 6.

Detection bias correction. 5C experiments involve a number of steps that can locally differ in efficiency, thereby introducing biases in efficiency of detection of pairs of interactions. These biases could be due to differences in the efficiency of crosslinking, the efficiency of restriction digestion (related to crosslinking efficiency), the efficiency of ligation (related to fragment size), the efficiency of $5 \mathrm{C}$ primers (related to annealing and PCR amplification) and finally the efficiency of DNA sequencing (related to base composition). All of these potential biasesseveral of which are common to other approaches such as chromatin immunoprecipitation (for example, crosslinking efficiency, PCR amplification, base-composition-dependent sequencing efficiency)-will have an impact on the overall efficiency with which long-range interactions for a given locus (restriction frag ment) can be detected. To determine this overall efficiency of interaction detection we have developed the following general strategy. To determine overall interaction detection efficiency for a given restriction fragment we analysed the large set of interchromosomal interactions that are detected for each fragment. We then defined the overall efficiency of interchromosomal interaction detection for a given fragment as the ratio of the average interchromosomal signal obtained with that fragment and the average interchromosomal signal of all fragments. We then corrected the frequency of each interrogated long-range intrachromosomal interaction using a correction factor that is the product of the overall efficiency of interchromosomal interaction detection for the two interacting fragments.

This procedure will correct for any of the biases in detectability of interactions for a given locus, as listed above, and will also adjust for copy number variation of a 
locus, which can vary in transformed cell lines such as K562 and HeLa-S3 cells, as these factors will also affect the level of interchromosomal interactions.

Detailed primer filtering. To approximate the relative $5 \mathrm{C}$ signal of each restriction fragment interrogated in the experiment we first calculated the average $5 \mathrm{C}$ signal for all trans interactions (interactions between different chromosomes). To remove any extreme outliers from the mean calculation (for example, due to primer failure) we first filtered down the distribution of 5C signals in trans for each restriction fragment by removing all signals beyond the mean \pm 3 standard deviations (s.d.). After calculating the filtered mean for each restriction fragment in trans, we calculated the global mean of all interchromosomal interaction frequencies. We then calculated a correction factor for each restriction fragment that would normalize its set of trans interactions to the entire set. Once the correction factors were calculated, we then calculated the mean and s.d. correction factor and flagged any restriction fragments requiring a correction value beyond the mean \pm 1.654 s.d. Fragments with a correction factor outside of this limit were flagged for removal as their trans signal is too above/below the expected signal by chance. Here, we assume that any variation in $5 \mathrm{C}$ signals detected within the trans space is due to experimental factors, differing primer efficiencies, ligation efficiencies, etc

Detailed primer correction. Once the outlier fragments are removed from the $5 \mathrm{C}$ data set, we repeated the above-described steps to calculate the primer correction values required to normalize the $5 \mathrm{C}$ signals for the remaining restriction fragments. Then, for each 5C interaction within an ENCODE region in the data set, we used the product of the correction factors from the two restrictions fragments involved in the interaction as the final correction factor to apply to the $5 \mathrm{C}$ signal. $5 \mathrm{C}$ signals were then either increased or decreased by the correction factor to correct for varying signals from the fragments visibility in the trans interaction space.

Peak calling. To detect significant looping interactions from background looping interactions we developed an in-house ' $5 \mathrm{C}$ peak calling' algorithm. We chose to call peaks in each $5 \mathrm{C}$ biological replicate separately and then take only the peaks that intersect across replicates as our final list of significant looping interactions.

$5 \mathrm{C}$ signals represent the three-dimensional contact probabilities between pairs of loci. This relationship inversely scaled with genomic distance. To control properly for the varying genomic distances tested in the $5 \mathrm{C}$ data set, we first determined the relationship of 5C signals over genomic distance. Using a Lowess smoothing algorithm we found the weighted average and weighted s.d. of all $5 \mathrm{C}$ signals across the range of all interrogated genomic distances. We used the traditional tri-cubic weighting function and an $\alpha$ parameter of 0.01 to average the closest $1 \%$ of the $5 \mathrm{C}$ signals around each genomic distance. We assumed that the large majority of interactions are not significant looping interactions and thus we interpreted this weighted average as the expected 5C signal for any given genomic distance. The $5 \mathrm{C}$ signals were then transformed into a $z$-score by calculating the (obs $-\exp / \mathrm{s}$.d.). Where the obs value is the detected $5 \mathrm{C}$ signal for a specific interaction, exp is the calculated weighted average of $5 \mathrm{C}$ signals for a specific genomic distance, and s.d. is the calculated weighted standard deviation of $5 \mathrm{C}$ signals for a specific genomic distance. Once the $z$-scores were calculated, the distribution of $z$-scores was fit to a Weibull distribution. We found that the distribution of $z$-scores fits to the Weibull distribution with an $R^{2}$ value of $>0.939$ for all cell lines. $P$ values can then be mapped to each $z$-score and then also transformed into $q$ values for FDR analysis. The ' $q$ value' package from $\mathrm{R}$ (qvalue.cal [siggenes]) was used to compute the $q$ values for the given set of $P$ values determined from the fit to the Weibull distribution. Using an FDR cutoff of $1 \%$, we selected all $5 \mathrm{C}$ interactions with a $q$ value $\leq 0.01$. We then took the intersection of all significant looping interactions across the two biological replicates as our final list of $5 \mathrm{C}$ looping interactions.

Estimation of frequency of false-positive looping interactions. We defined a false-positive $5 \mathrm{C}$ looping interaction as an interaction that is identified in the peak calling approach described above but is due to technical biases or noise and thus does not reflect a biologically meaningful long-range interaction. To estimate the frequency by which our approach detects significant looping interactions by chance, we analysed 5C data obtained for the three ENCODE regions that are devoid of genes and are almost devoid of active regulatory elements (according the ENCODE seven-way segmentation ${ }^{4}$ ). As described above, we used an alternating $5 \mathrm{C}$ primer design for these regions. As a result, long-range interaction profiles are not specifically anchored on any type of genomic element. Combined with the fact that these regions are largely devoid of any functional elements, we do not expect to detect any significant looping interactions. Thus, assessment of the number of looping interactions detected for these regions using our peak-calling pipeline provides an empirical approach to estimate the frequency by which significant looping interactions are detected by chance and thus represent false positives.

Supplementary Fig. 1a shows the number of peaks detected in the three gene desert ENCODE regions (ENr112, ENr113 and ENr313). We used these numbers to estimate the frequency with which we detect significant looping interactions by chance. For GM12878 cells we identified 17 significant looping interactions in both replicates. For these three ENCODE regions we interrogated 7,819 5C interactions. Thus, we estimate that the fraction of interrogated interactions that by chance scores as a significant long-range interaction: $(17 / 7,819) 100=0.217 \%$. Assuming that this fraction is the same for the set of 82,545 interrogated TSSdistal element interactions throughout the ENCODE regions, we expect to detect $(0.217 \times 82,545) / 100=179$ false-positive looping interactions. We detected 1,011 significant looping interactions between TSSs and distal sites in GM12878 cells, which leads us to estimate that the false-positive detection rate is around $18 \%$ $[(179 / 1,011) 100]$. Similar analyses of 5C data from K562 and HeLa-S3 cells lead to estimates of false-positive detection rates of $10 \%$ and $12 \%$, respectively, corresponding to 147 out of 1,434 and 190 out of 1,620 looping interactions possibly being false positives. We note that these represent upper limit estimates, as some of the significant looping interactions detected in the gene desert regions may be real.

The false-positive detection rate for single replicates can be calculated in exactly the same way. We found that the fraction of significant looping interactions detected in one replicate that might be false positives ranges from $20 \%$ to $47 \%$. Thus, by requiring interactions to be significant in both replicates, we greatly reduce the fraction of false-positive significant interactions (from $20-47 \%$ to $10-18 \%$ of the significant interactions). At the same time, many of the significant interactions detected in only one replicate were not false positives, and by excluding this subset of interactions from our analysis we introduce false negatives. Consistent with our interpretation that many of the peaks seen in only one replicate represent false negatives, we found that when we take the union of the peaks found in replicates 1 and 2, or analyse the set of peaks obtained with individual replicates separately, all of the results that we presented remain the same: (1) enrichment for distal elements that resemble active gene regulatory elements (Supplementary Fig. 1e); (2) asymmetry of the long-range interaction landscape with a peak around $120 \mathrm{~kb}$ upstream of the TSS (Supplementary Fig. 8); (3) skipping over CTCF sites; and (4) formation of interwoven interaction networks. The fact that all our results can be obtained using different peak sets (for example, the union of two replicates, or the intersection of the replicates) indicates that our basic findings are robust and not very sensitive to where the threshold for peaks is placed. By focusing exclusively on the set of peaks independently detected in both replicates we are being conservative, only report the strongest signals that display the strongest enrichments for active chromatin features (Supplementary Fig. 1), and reduce the false-positive rate.

In general we prefer false negatives over false positives.

Fragment annotation. To annotate the interrogated restriction fragments, a variety of ENCODE data sets were used to check for overlap with our list of restriction fragments. A list of all used ENCODE data sets can be found in Supplementary Table 7.

Supplementary data. A zip archive containing all Supplementary Data can be found in Supplementary Information.

29. Dostie, J. \& Dekker, J. Mapping networks of physical interactions between genomic elements using 5C technology. Nature Protocols 2, 988-1002 (2007), 